\title{
The Effect of Herbal Medicine Supplementation on Clinical and Para-clinical Outcomes in Women With PCOS: A Systematic Review and Meta-analysis
}

\author{
Nava Ainehchi ${ }^{\mathbb{D}}$, Azizeh Farshbaf-Khalili' ${ }^{2}$, Aliyeh Ghasemzadeh ${ }^{1}$, Kobra Hamdi ${ }^{1}$, Arash Khaki ${ }^{1}$, Elaheh \\ Ouladsahebmadarek $^{1^{*} \mathbb{D}}$, Abbas Delazar $^{3}$, Fahimeh Bakhtyari ${ }^{2}$, Masoumeh Mazandarani ${ }^{4}$
}

\begin{abstract}
Objectives: The aim of this study is to assess the impact of Cinnamomum verum, Mentha spicata, Zingiberene officinal on polycystic ovary syndrome (PCOS) treatment.

Materials and Methods: MEDLINE, Cochrane Central Register of Controlled Trials (CENTRAL), EMBASE, Scopus, Web of Science, Google Scholar, ProQuest, Clinicaltrial.gov, and for Persian articles SID, Magiran, Irandoc, and Iranmedex were searched without any time limitation.

Results: Thirteen randomized controlled trials (RCTs) consisting 668 women were entered in the meta-analysis. Significant differences in fasting blood sugar (FBS; weighted mean difference (WMD) $=-3.69 \mathrm{mg} / \mathrm{dL}, 95 \% \mathrm{CI}:-6.67$ to $-0.7, P=0.02 ; 241$ participants), fasting insulin (WMD $=-4.53 \mu \mathrm{IU} / \mathrm{mL}, 95 \% \mathrm{CI}:-6.45$ to $-2.61, P<0.001 ; 183$ participants), triglyceride (TG; WMD $=-17.97 \mathrm{mg} /$ dL, $95 \%$ CI: -30.51 to $-5.43, P=0.005 ; 183$ participants), total cholesterol (TC; WMD=-14.60 mg/dL, 95\% CI: -22.93 to -6.26 , $P=0.0006 ; 183$ participants), low-density lipoprotein cholesterol (LDL; WMD $=-16.58 \mathrm{mg} / \mathrm{dL}, 95 \%$ CI -23.91 to $-9.24, P<0.001 ; 183$ participants), malondialdehyde (MDA; WMD $=-0.25 \mathrm{nmol} / \mathrm{ml}, 95 \% \mathrm{CI}-0.41$ to $-0.09, P<0.002 ; 124$ participants), total testosterone (TT; WMD $=-0.18 \mathrm{ng} / \mathrm{mL}, 95 \%$ CI -0.27 to $-0.09, P<0.001 ; 116$ participants), free testosterone (FT; WMD $=-5.47 \mathrm{pg} / \mathrm{mL}, 95 \% \mathrm{CI}$ -8.34 to $-2.61, P=0.0002 ; 78$ participants) were obtained by using cinnamon alone and herbal mixture containing cinnamon in comparison to control.

Conclusions: This meta-analysis showed that cinnamon alone and herbal mixture containing cinnamon improve level of FBS, fasting insulin, TG, TC, LDL, MDA, TT, and FT serum level.

Keywords: Polycystic ovary syndrome, Zingiber officinale, Mentha spicata, Cinnamomum zeylanicum Nees, Medicinal herb
\end{abstract}

\section{Introduction}

Polycystic ovary syndrome (PCOS) is the most common endocrine disorder among women of child-bearing age that affects $6 \%-26 \%$ of reproductive age population (1). The most common symptoms of this syndrome include elevated androgens, irregular or no menstrual cycles, hirsutism, insulin resistance and difficulty getting pregnant (2). Associated conditions include type 2 diabetes, obesity, heart diseases, and endometrial cancer. Although insulin resistance is not criteria for the PCOS diagnosis, the prevalence is $50 \%-70 \%$ (3). Hyperinsulinemia could contribute to PCOS pathogenesis by elevating androgen secretion, disrupting menstrual cyclicity and folliculogenesis (4). The relationships between sex steroids and the hypothalamic gonadotropin releasing hormone (GnRH) modulated level of physiological gonadotropin (5). In women with PCOS, higher GnRH pulse frequencies lead to increase secretion of

Luteinizing hormone (LH) to follicle-stimulating hormone (FSH) secretion. As a consequence, the enhanced pituitary-released LH secretion and reduced FSH not only contribute to impaired folliculogenesis, but also seem to cause androgen over production by follicular theca cells whereas lower FSH levels cause to anovulation (6).

On the other hand, studies declare that reactive oxygen species (ROS) has been associated with PCOS, oxidative stress (OS) in excess level damage cellular functions and by influencing ovulation can affect female fertility (7). Also, OS products exposed to islets, impaired insulin secretion and lead to glucose oxidation (8).

Cinnamomum zeylanicum Nees (Cinnamon) from the Lauraceae family has been known for its antioxidant and anti-inflammatory properties and by phenolic content particularly cinnamaldehyde as an antioxidant, improves serum level of superoxide dismutase (SOD), catalase (CAT), glutathione peroxidase (GPX), and decreases malondialdehyde (MDA) level, elevated rate of pregnancy (9). It also decrease level of fasting blood sugar (FBS) and insulin as well as, total cholesterol (TC), low-density lipoprotein (LDL), and triglyceride (TG) (10).

Zingiber officinale Roscoe (ginger) from Zingiberaceae family reduces serum levels of FBS, insulin and LDL-C/ 
HDL-C (11). Some studies have shown that ginger elevates the antioxidant capacity in blood, as well as enhance the serum levels of SOD, CAT, and GPX (12).

Mentha spicata (spearmint) from Lamiaceae family, widely spread in the temperate zone, has antiinflammatory, anti-diabetic, and decreases testosterone production, has anti-androgen properties, improves hirsutism and has anti-inflammatory, anti-diabetic, and anticancer virtues (13). Moreover, spearmint improves ovarian cysts in PCOS by reducing atretic follicles, and enhancing graafian follicles (14).

The purpose of the present systematic review and meta-analysis was to appraise the outcomes from published randomized controlled trials (RCTs) and quasi experimental studies and investigate the impact of herbal medicine supplementation on clinical and paraclinical outcomes in women affected by PCOS. We also appraised the hypothesis "potency of herbal medicine supplementation in PCOS women has beneficial effects." Similar studies have been published (15-17). However, authors aimed to omit the irrelevant data and perform it in a strictly focused way.

\section{Materials and Methods}

\section{Search Strategies and Information Sources}

The present study has been designed based on Cochrane Collaboration handbook guidelines (18) and Techniques and Guides for Systematic Reviews and Meta-Analyses: the PRISMA statement $(19,20)$. The primary aim of this systematic review and meta-analysis was to examine the para-clinical outcomes (FBS, insulin, homeostasis model assessment-insulin resistance [HOMA-IR], quantitative insulin sensitivity check index [QUICKI], TG, TC, LDL-C, HDL-C, very low-density lipoprotein [VLDL], $\mathrm{FSH}$, LH, free testosterone [FT], total testosterone [TT], estradiol [E2], MDA, SOD, GPx, CAT, thyroidstimulating hormone [TSH], free thyroxine [FT4], free triiodothyronine [FT3]) and clinical outcomes (like body weight, body mass index [BMI], hirsutism, acne, oligomenorrhea, amenorrhea, menstrual regulation, and the rate of pregnancy) were considered as a secondary outcome. Two authors (N.A. and A.F-Kh.) systematically searched literature using electronic database including MEDLINE (PubMed), Cochrane Central Register of Controlled Trials (CENTRAL), EMBASE, Scopus, Web of Science, Google Scholar, ProQuest, Clinicaltrial.gov, and for Persian articles SID, Magiran, Irandoc, and Iranmedex were used based on title and abstract without any time limitation (Figure 1). For literature extraction, $\mathrm{MeSH}$ terms from online source were designed as following: ("polycystic ovarian syndrome" OR "polycystic ovary syndrome" OR "Polycystic Ovary Syndrome 1" OR "PCOS" OR "polycystic ovary disease" OR "Sclerocystic Ovarian Degeneration" OR "ovary sclerocystic" OR "sclerocystic ovary syndrome" OR "Sclerocystic Ovaries" OR "Stein Leventhal Syndrome") AND ("Zingiber officinale" OR "Ginger") AND ("Cinnamomum verum" OR "Cinnamomum zeylanicum" OR "cinnamon*” OR "Cinnamomin") AND ("Mentha spicata" OR "spearmint”) AND ("herb*").

It is worth noting that the references of all obtained articles were searched as well to identify any additional
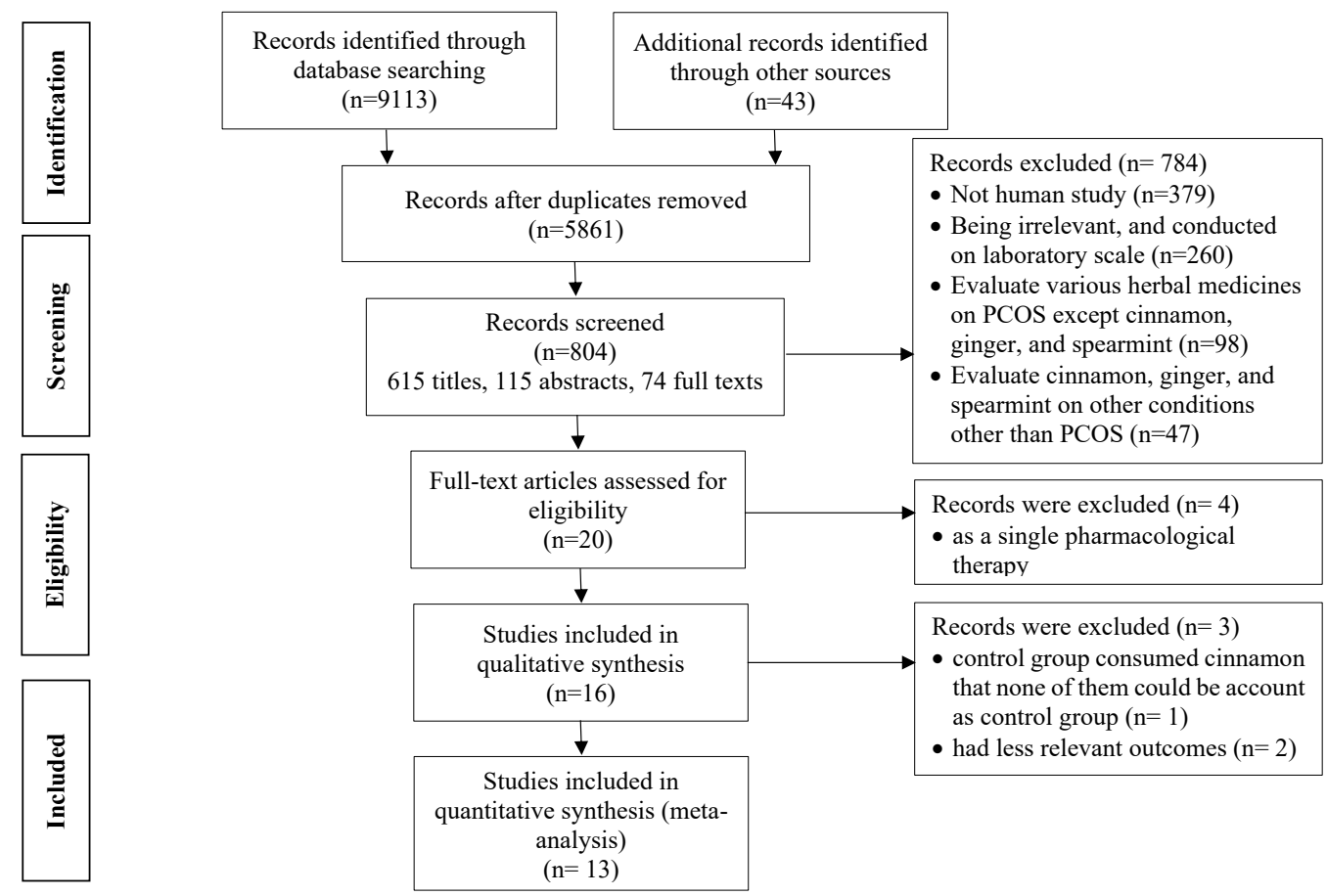

Figure 1. Diagram for the Search and Selection Process of Articles. 
relevant studies on the same topic.

After the initial search, 2 authors in terms of publishing new articles related to the purpose of this study conducted searching via regular weekly searches of CENTRAL, Scopus, EMBASE, MEDLINE (PubMed), Web of Science, Google Scholar, ProQuest as well as Persian databases such as Iranmedex, SID, Irandoc, and Magiran.

EndNote (version X7) (Philadelphia, PA) was employed to manage and store the references by the mentioned strategies.

\section{Eligibility Criteria}

To identify eligible trials, 3 researchers (N.A., A.F-Kh., and A.Kh.) independently screened titles, abstracts, and full text of selected papers in detail. For inclusion, the relevant studies were separately reviewed by (N.A., A.F. Kh., and A.Kh) to omit duplicate cases. Then the potentially qualified full text for citations was evaluated by two of the reviewers. In the case of disagreements on qualification, these were resolved by discussion with the third author. The PICOS format and search keywords (Participants, Interventions, Comparators, Outcomes, and Study design) was used to examine the study eligibility criteria (Table 1).

\section{Inclusion Criteria}

All quasi-experimental and RCTs studies that investigated cinnamon, spearmint, ginger lonely or in combination with other herbs which were compared with placebo or control as standard treatment in PCOS patient were included in this review and meta-analysis. All English and Persian language studies were included and chosen studies investigate the effect of herbal medicine supplementation on various indices like body weight, BMI, FBS, insulin, HOMA-IR, QUICKI, TG, TC, LDL-C, HDL-C, VLDL, FSH, LH, FT, TT, E2, MDA, SOD, GPx, CAT, TSH, FT4, FT3, hirsutism, acne, oligomenorrhea, amenorrhea, menstrual regulation, and the rate of pregnancy in PCOS women.

\section{Exclusion Criteria}

Semi-experimental articles, review studies, animal studies, study protocols, letters, and trials without a control arm were not incorporated.

A total of 9156 free full text citations were detected. After title, abstract, and full text evaluation, 20 eligible studies were retrieved for full text revision. Four of studies based on mentioned eligibility criteria in Figure 1 were excluded. Finally, 16 studies met the selection criteria

\section{Data Extraction}

Including the data from all eligible articles that contained the following elements: Author name (year), type of study, sample size, sex, drug, country, participant age, intervention (dosage and frequency), comparison (dosage and frequency), duration of follow-up, outcomes (primary /secondary), method of measurements, results, side effects. Any disagreements for screening among authors were resolved by consultation with other author (F.B.).

\section{Risk of Bias Assessment}

The judgment for parallel group trials involves assessing the random sequence generation (selection bias), allocation concealment (selection bias), masking of participants and personnel (performance bias), masking of outcome assessment (detection bias), incomplete outcome data (attrition bias), and selective reporting (reporting bias) by 2 authors (N.A. and A.F-Kh.) based on Cochrane Collaboration's tool (21).

Any disagreements were solved by consulting the fifth author (A.Kh.). Risk of bias is evaluated as low, high, or unclear.

Data Synthesis and Analysis

To perform meta-analysis, Review Manager (RevMan v5.3, The Cochrane Collaboration, Oxford, UK) were used. Mean differences (MD) were calculated with $95 \%$ confidence intervals (CIs) based on intention-to-treat analysis for all included papers if possible. Otherwise, available data were used. The mean \pm SD were calculated for all outcomes. If not available, they were obtained from other dispersion measures and central tendency reported in article like median and interquartile range (18).

Table 1. PICOS Criteria for Inclusion and Exclusion of Studies

\begin{tabular}{ll}
\hline Parameter & Determined criteria for present study \\
\hline Participants & $\begin{array}{l}\text { Women aged 12-42 years who were treated with Cinnamomum zeylanicum Nees, or Mentha spicata, or Zingiberene officinale } \\
\text { lonely or in combination with other herbs for polycystic ovary syndrome }\end{array}$ \\
\hline Intervention & $\begin{array}{l}\text { Cinnamomum zeylanicum Nees, or Mentha spicata, or Zingiberene officinale without restrictions regarding dose (e.g., high, } \\
\text { low), dosing interval (e.g., daily, weekly), and pharmaceutical form (e.g., extract or powder in capsule, tea) }\end{array}$ \\
\hline Comparator & Placebo or control (metformin, clomiphene citrate, wheat flour, starch) \\
Outcomes & $\begin{array}{l}\text { Any beneficial effect of Cinnamomum zeylanicum Nees, or Mentha spicata, or Zingiberene officinale supplementation on } \\
\text { serum antioxidants (CAT, GPX, SOD, MDA), glycemic biomarkers (insulin, insulin resistance, and FBS), sex steroids (FSH, } \\
\text { anthropometric indices (weight, BMI, waist circumference, waist to hip), clinical features (hirsutism, acne, oligomenorrhea, } \\
\text { amenorrhea, menstrual regulation, and the rate of pregnancy), thyroid hormones (FT4, FT3, TSH) }\end{array}$ \\
\hline Study design & RCTs or quasi-experimental studies.
\end{tabular}


Moreover, according to $\mathrm{SD}=\mathrm{SEM} *$ sqrt $(\mathrm{N})$ equation, SEM convert to SD. The changes of the mean (SD) of all continuous variables were used for computing MDs with 95\% confidence intervals (CIs). In studies that reported only the (means \pm SDs) in the baseline and follow-up measurements for each group, the means and SDs for change in the outcome were computing for the study groups. The mean changes for continuous outcome as the follow-up period minus measurement at baseline. Change score of SD was calculated as:

$$
S D_{\text {change score }}=\sqrt{S D_{b}^{2}+S D_{f}^{2}-2 * r * S D_{b} * S D_{f}}
$$

$\mathrm{SD}_{\mathrm{b}}$ : $\mathrm{SD}$ at baseline, $\mathrm{SD}_{\mathrm{f}} \mathrm{SD}$ at follow-up, and $\mathrm{r}$ : correlation between baseline and the follow-up score (22). The statistical heterogeneity across trials were assessed by $\mathrm{I}^{2}$ statistic, which describes the percentage of variation, and value of $\mathrm{I}^{2}$ greater than $50 \%$ was considered heterogeneous. All results were presented as random-effect model when the detected heterogeneity was significant. Otherwise, a fixed-effect model was used for minimal heterogeneity. Result of this study. Forest plot displayed estimated results.

\section{Results}

Literature Search

The details and reasons for study selection and exclusion of research are shown in figure 1. 16 RCTs met the inclusion criteria and were obtained for fulltext detail review. Upon further scrutiny, 1 of 16 RCTs, which had less relevant outcomes (23) and one of them did not include original data (24) and the last one consumed cinnamon in both control and intervention group (25) were excluded from meta-analysis.

\section{Selected Articles}

A total of 9156 articles were identified; of these, 5861 researches were excluded as duplicate and 804 relevant papers were screened for inclusion. Then, 784 of them were excluded as not clinical trial research, irrelevant, conducting on laboratory scale, evaluating various herbal medicines except cinnamon, ginger, and spearmint on PCOS, or investigating cinnamon, ginger, and spearmint effectiveness on other disorders (except PCOS). Finally, out of 16 articles, 13 were included in the meta-analysis, and 3 (23-25) were reviewed (Figure 1).

\section{Description of Studies}

The included RCTs and quasi-experimental papers were published from 2007-2019. From 16 articles, 15 were written in English and 1 in Persian. Eight articles measured cinnamon efficacy on PCOS alone (24,26-32), and another 8 articles measured herbal mixture that all of them contain cinnamon along with other herbs like spearmint, ginger, Glycyrrhiza glabra, etc (15,16,25,33-35); just one RCT evaluated spearmint effect in PCOS women
(13). Studies were conducted in United States, Australia, the United Kingdom, Japan, Iran, India, Indonesia, and Egypt. There was considerable methodological and clinical heterogeneity among studies with respect to study length and dose of supplement. The details of these studies are summarized in Table S1-S3 (See Supplementary file 1).

Risk of Bias in Included Studies

The risk of bias rating for each included study was evaluated according to researchers' decisions which are shown in Figures $2 \& 3$ and Table 2.

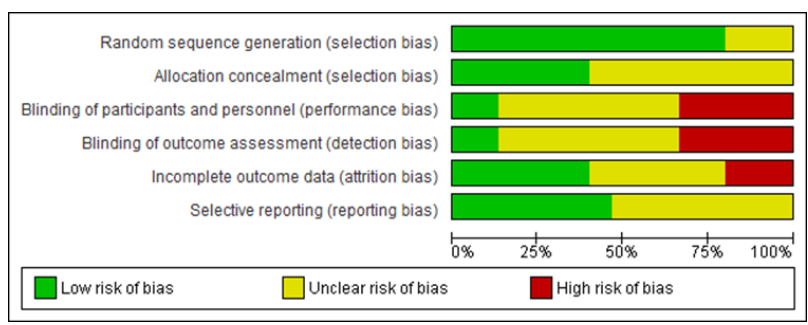

Figure 2. Risk of Bias Graph: review authors' judgments about each risk of bias item presented as percentages across all included studies.

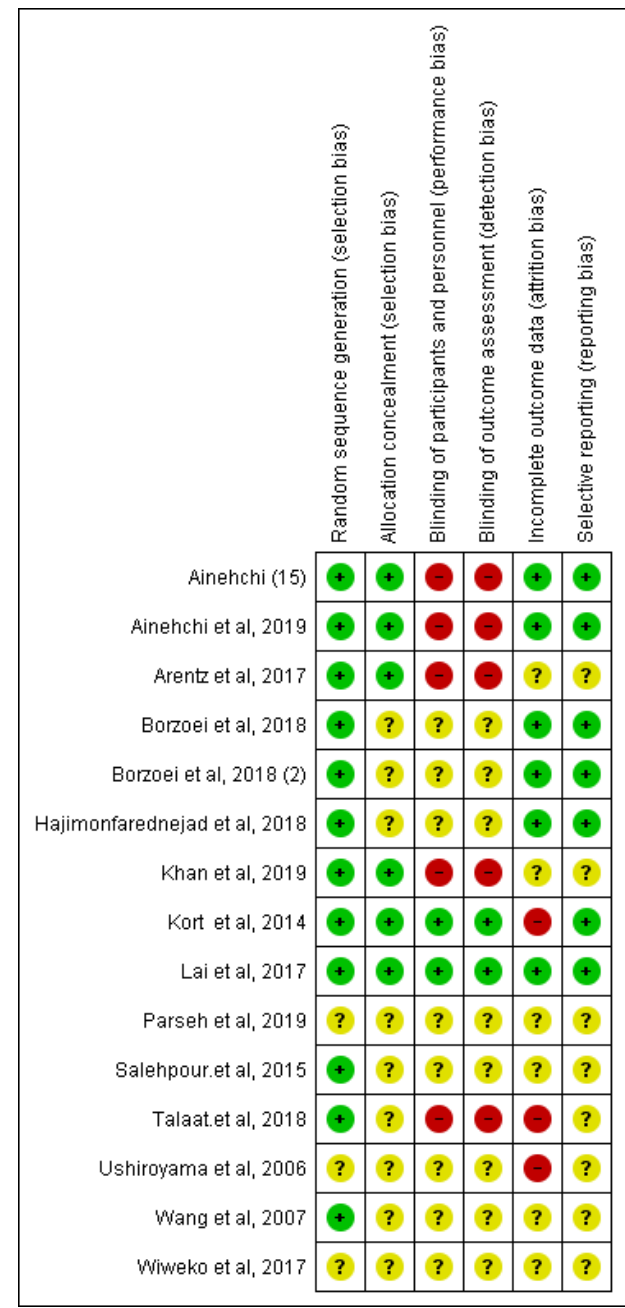

Figure 3. Diagram of Bias in the Included Studies. 
Efficacy of Intervention

Effect of cinnamon alone and herbal mixture containing cinnamon and other herbs versus placebo or control on BMI Seven trials $(16,26,27,31,33-35)$ with 538 participants evaluated the effect of cinnamon alone and herbal mixture versus placebo or control on BMI level. As the $\mathrm{I}^{2}$ of $89 \%$ represents significant heterogeneity among the studies $(P<0.001)$, a random effect model was applied to pool outcomes. The calculated overall effect size illustrated that the differences between 2 groups was not statistically significant $\left(\mathrm{MD}=-1.1 \mathrm{~kg} / \mathrm{m}^{2}, 95 \% \mathrm{CI}:-2.7\right.$ to $0.49, P=$ 0.18 ; Figure S1a).

Subgroup analysis of BMI to evaluate the efficacy of cinnamon alone versus placebo

Three trials $(26,27,31)$ with 163 participants just studied effect of cinnamon alone versus placebo, and studies of herbal mixture and groups consumed metformin were excluded. As the $\mathrm{I}^{2}$ of $0 \%$ represents no statistic heterogeneity among the studies $(P=0.84)$, a fixed effect model was applied to pool the outcomes. The obtained overall effect size illustrated a statistically significant difference in the BMI level in PCOS women consumed cinnamon supplement, meaning that take of cinnamon supplements in women suffer from PCOS led to a significant lower BMI level $\left(\mathrm{MD}=-0.69 \mathrm{~kg} / \mathrm{m}^{2}, 95 \% \mathrm{CI}\right.$ : -1.03 to $-0.35, P<0.001$; Figure $S 1 b)$.

Effect of cinnamon alone and herbal mixture containing cinnamon and other herbs versus placebo or control on weight

Three trials $(27,30,34)$ with 265 participants evaluated the effect of cinnamon alone and herbal mixture versus placebo or control on weight. As the $\mathrm{I}^{2}$ of $0 \%$ represents no statistic heterogeneity among the studies $(P=0.87)$, a fixed effect model was applied to pool the outcomes. The calculated overall effect size illustrated that the differences between two study groups was not statistically significant
$(\mathrm{MD}=-1.22 \mathrm{~kg}, 95 \% \mathrm{CI}:-4.61$ to $2.16, P=0.48$; Figure S1c).

Effect of cinnamon alone and herbal mixture containing cinnamon and other herbs versus placebo or control on waist circumference

Two trials $(27,34)$ with 181 participants evaluated the effect of cinnamon alone and herbal mixture versus placebo or control on waist circumference. As the $\mathrm{I}^{2}$ of $0 \%$ represents no statistic heterogeneity among the studies $(P=0.72)$, a fixed effect model was applied to pool the outcomes. The estimated overall effect size illustrated that the differences between two study groups was not significant $(\mathrm{MD}=$ $-2.53 \mathrm{~cm}, 95 \% \mathrm{CI}:-6.47$ to $1.41, P=0.21$; Figure $\mathrm{S} 1 \mathrm{~d}$ ).

Effect of cinnamon alone and herbal mixture containing cinnamon and other herbs versus placebo or control on FBS Five trials $(16,27,29-31)$ with 241 participants evaluated the effect of cinnamon alone and herbal mixture versus placebo or control on FBS level. As the $\mathrm{I}^{2}$ of $54 \%$ represents high heterogeneity among the studies $(P=0.07)$, a random effect model was applied to pool outcomes. The estimated overall effect size demonstrated a statistically significant diverse in the FBS level in PCOS women taking cinnamon alone and herbal mixture supplement, meaning that cinnamon and herbal mixture supplementation had a significant decrease in the FBS level $(\mathrm{MD}=-3.69 \mathrm{mg} / \mathrm{dL}$, 95\% CI: -6.67 to $-0.7, P=0.02$; Figure S1e).

Subgroup analysis of FBS to evaluate the efficacy of cinnamon alone versus placebo

Three trials $(27,30,31)$ with 163 participants just studied effect of cinnamon alone versus placebo, and studies of herbal mixture and groups consumed metformin were excluded. As the $\mathrm{I}^{2}$ of $67 \%$ represents high heterogeneity among the studies $(P=0.05)$, a random effect model was applied to pool outcomes. The obtained overall effect size illustrated a statistically meaningful diversion in the

Table 2. Risk of Bias in RCTs and Quasi-experimental Studies According to the Cochrane Collaboration's Tool for Assessing the Risk of Bias in Randomised Clinical Trials

\begin{tabular}{|c|c|c|c|c|c|c|c|c|c|c|c|c|c|c|c|c|}
\hline Bias risk & (26) & (27) & (28) & (24) & (29) & (30) & (31) & (32) & (33) & (34) & (25) & (23) & (35) & (16) & (15) & (13) \\
\hline $\begin{array}{l}\text { Random sequence } \\
\text { generation (selection bias) }\end{array}$ & 1 & 1 & 1 & 1 & 1 & 1 & 3 & 1 & 3 & 1 & 1 & 3 & 1 & 1 & 1 & 1 \\
\hline $\begin{array}{l}\text { Allocation concealment } \\
\text { (selection bias) }\end{array}$ & 3 & 3 & 1 & 3 & 1 & 3 & 3 & 3 & 3 & 1 & 1 & 3 & 3 & 1 & 1 & 3 \\
\hline $\begin{array}{l}\text { Blinding of participants and } \\
\text { personnel (performance bias) }\end{array}$ & 3 & 3 & 1 & 3 & 2 & 3 & 3 & 3 & 3 & 2 & 1 & 3 & 2 & 2 & 2 & 2 \\
\hline $\begin{array}{l}\text { Blinding of outcome } \\
\text { assessment (detection bias) }\end{array}$ & 3 & 3 & 1 & 3 & 2 & 3 & 3 & 3 & 3 & 2 & 1 & 3 & 2 & 2 & 2 & 2 \\
\hline $\begin{array}{l}\text { Incomplete outcome data } \\
\text { (attrition bias) }\end{array}$ & 1 & 1 & 2 & 3 & 3 & 1 & 3 & 3 & 3 & 3 & 1 & 2 & 2 & 1 & 1 & 3 \\
\hline $\begin{array}{l}\text { Selective reporting (reporting } \\
\text { bias) }\end{array}$ & 1 & 1 & 1 & 3 & 3 & 1 & 3 & 3 & 3 & 3 & 1 & 3 & 3 & 1 & 1 & 3 \\
\hline
\end{tabular}

Note. 1: Low risk (+); 2: High risk (-); 3: unknown risk. 
FBS level in PCOS women taking cinnamon supplement, meaning that intake of cinnamon supplements in women with PCOS caused a significant lower FBS level $(\mathrm{MD}=$ $-5.17 \mathrm{mg} / \mathrm{dL}, 95 \% \mathrm{CI}$ : -9.75 to $-0.58, P=0.03$; Figure $\mathrm{S} 1 \mathrm{f}$ ).

Effect of cinnamon alone and herbal mixture containing cinnamon and other herbs versus placebo or control on HOMA-IR

Six trials $(16,27,28,0-32)$ with 295 participants evaluated the effect of cinnamon alone and herbal mixture versus placebo or control on HOMA-IR level. As the $\mathrm{I}^{2}$ of $96 \%$ represents high heterogeneity between the studies $(P$ $<0.001$ ), a random effect model was applied to pool outcomes. The obtained overall effect size indicated that the diversity between two study groups was not statistically meaningful $(\mathrm{MD}=-0.8,95 \% \mathrm{CI}:-1.74$ to $0.13, P=0.09$; Figure S1g).

Subgroup analysis of HOMA-IR to evaluate the efficacy of cinnamon alone versus placebo

Five trials $(27,28,30-32)$ with 255 participants just studied effect of cinnamon alone versus placebo, and one study of herbal mixture (16) was excluded. As the $\mathrm{I}^{2}$ of $96 \%$ represents high heterogeneity among the studies $(P<0.001)$, a random effect model was applied to pool outcomes. The calculated overall effect size illustrated that the differences between two study groups was not meaningful $(\mathrm{MD}=-0.77,95 \% \mathrm{CI}:-1.84$ to $0.30, \mathrm{p}=0.16$; Figure S1h).

Effect of cinnamon alone and herbal mixture containing cinnamon and other herbs versus placebo or control on fasting insulin

Three trials $(16,27,30)$ with 183 participants evaluated the effect of cinnamon alone and herbal mixture versus placebo or control on fasting insulin level. As the $\mathrm{I}^{2}$ of $0 \%$ represents no statistic heterogeneity among the studies $(P=0.57)$, a fixed effect model was applied to pool the outcomes. The obtained overall effect size illustrated a statistically meaningful diversity in the fasting insulin level in PCOS women taking cinnamon alone and herbal mixture supplement, meaning that taking cinnamon alone or as herbal mixture supplements in females with PCOS caused a significant lower fasting insulin level $(\mathrm{MD}=-4.53$ $\mu \mathrm{IU} / \mathrm{mL}, 95 \% \mathrm{CI}:-6.45$ to $-2.61, P<0.001$; Figure S1i).

Effect of cinnamon alone and herbal mixture containing cinnamon and other herbs versus placebo or control on TG Three trials $(15,26,27)$ with 183 participants evaluated the effect of cinnamon alone and herbal mixture versus placebo or control on TG level. As the $\mathrm{I}^{2}$ of $24 \%$ represents no statistic heterogeneity among the trials $(P=0.27)$, a fixed effect model was applied to pool the outcomes. The obtained overall effect size illustrated a statistically significant diversity in the TG level in PCOS women taking cinnamon alone and herbal mixture supplement, meaning that consumption of cinnamon alone and herbal mixture supplementation in women suffering from PCOS caused a significant lower TG level $(\mathrm{MD}=-17.97 \mathrm{mg} / \mathrm{dL}$, 95\% CI: -30.51 to $-5.43, \mathrm{p}=0.005$; Figure $\mathrm{S} 1 \mathrm{j}$ ).

Effect of cinnamon alone and herbal mixture containing cinnamon and other herbs versus placebo or control on TC Three trials $(15,26,27)$ with 183 participants evaluated the effect of cinnamon alone and herbal mixture versus placebo or control on TG level. As the $\mathrm{I}^{2}$ of $0 \%$ represents no statistic heterogeneity among the studies $(P=0.58)$, a fixed effect model was applied to pool the outcomes. The calculated overall effect size illustrated a diversity in the TC level in PCOS women taking cinnamon alone and herbal mixture supplementation, meaning that taking cinnamon alone and herbal mixture supplementation in females with PCOS caused a significant lower TC level $(\mathrm{MD}=-14.60 \mathrm{mg} / \mathrm{dL}, 95 \% \mathrm{CI}:-22.93$ to $-6.26, P=0.0006$; Figure S1k).

Effect of cinnamon alone and herbal mixture containing cinnamon and other herbs versus placebo or control on LDL Three trials $(15,26,27)$ with 183 participants evaluated the effect of cinnamon alone and herbal mixture versus placebo or control on LDL level. As the $\mathrm{I}^{2}$ of $17 \%$ represents no statistic heterogeneity among the studies $(P=0.30)$, a fixed effect model was applied to pool the outcomes. The obtained overall effect size illustrated a statistically meaningful diversity in the LDL level in PCOS women consumed cinnamon alone and herbal mixture supplementation, meaning that consumption of cinnamon alone and herbal mixture in women suffer from PCOS caused a significant lower LDL level (MD $=-16.58$ mg/dL, 95\% CI: -23.91 to $-9.24, P<0.001$; Figure S1I).

Effect of cinnamon alone and herbal mixture containing cinnamon and other herbs versus placebo or control on HDL Three trials $(15,26,27)$ with 183 participants evaluated the effect of cinnamon alone and herbal mixture versus placebo or control on HDL level. As the $\mathrm{I}^{2}$ of $77 \%$ represents high heterogeneity among the studies $(P=0.01)$, a random effect model was applied to pool outcomes. The obtained overall effect size illustrated that the differences between 2 groups was not statistically significant $(\mathrm{MD}=6.58 \mathrm{mg} / \mathrm{dL}$, 95\% CI: -0.39 to $13.55, P=0.06$; Figure S1m).

Effect of cinnamon alone and herbal mixture containing cinnamon and other herbs versus placebo or control on $M D A$

Two trials $(16,26)$ with 124 participants evaluated the effect of cinnamon alone and herbal mixture versus placebo or control on MDA level. As the $\mathrm{I}^{2}$ of $0 \%$ represents no statistic heterogeneity among the studies $(P=0.33)$, a fixed effect model was applied to pool the outcomes. The obtained overall effect size demonstrated a statistically significant difference in the MDA level in PCOS women 
taking cinnamon alone and herbal mixture supplement, meaning that supplementation with cinnamon alone and herbal mixture in women suffer from PCOS led to a significant lower MDA level $(\mathrm{MD}=-0.25 \mathrm{nmol} / \mathrm{mL}, 95 \%$ CI: -0.41 to $-0.09, P<0.002$; Figure $\mathrm{S} 1 \mathrm{n}$ ).

Effect of herbal mixture containing cinnamon and other herbs versus placebo or control on FSH

Two trials $(15,35)$ With 215 participants evaluated the effect of herbal mixture versus placebo or control on FSH level. As the $\mathrm{I}^{2}$ of $0 \%$ represents no statistic heterogeneity between the studies $(P=0.36)$, a fixed effect model was applied to pool the outcomes. The obtained overall effect size illustrated that the differences between 2 groups was not significant $(\mathrm{MD}=-0.07 \mathrm{mIU} / \mathrm{mL}, 95 \% \mathrm{CI}$ : -0.47 to $0.34, P=0.75$; Figure $\mathrm{S} 1 \mathrm{o}$ ).

Effect of herbal mixture containing cinnamon and other herbs versus placebo or control on $\mathrm{LH}$

Two trials $(15,35)$ with 215 participants evaluated the effect of herbal mixture versus placebo or control on $\mathrm{LH}$ level. As the $\mathrm{I}^{2}$ of $0 \%$ represents no statistic heterogeneity between the studies $(P=0.50)$, a fixed effect model was applied to pool the results. The estimated overall effect size demonstrated that the differences between 2 groups was not statistically significant $(\mathrm{MD}=-0.61 \mathrm{mIU} / \mathrm{mL}, 95 \%$ CI: -1.24 to $0.01, P=0.05$; Figure $S 1 p$ ).

Effect of cinnamon alone and herbal mixture containing cinnamon and other herbs versus placebo or control on TT Three trials $(15,27,28)$ with 116 participants evaluated the effect of cinnamon alone and herbal mixture versus placebo or control on TT level. As the $\mathrm{I}^{2}$ of $0 \%$ represents no statistic heterogeneity among the studies $(P=0.56)$, a fixed effect model was applied to pool the outcomes. The obtained overall effect size illustrated a significant difference in the TT level in PCOS women taking cinnamon alone and herbal mixture supplementation, meaning that supplementation with cinnamon alone and herbal mixture in PCOS patients led to a significant lower TT level $(\mathrm{MD}=-0.18 \mathrm{ng} / \mathrm{mL}, 95 \% \mathrm{CI}:-0.27$ to $-0.09, P<$ 0.001; Figure S1q).

Effect of cinnamon alone and herbal mixture containing cinnamon and other herbs versus placebo or control on FT Two trials $(15,29) 78$ participants evaluated the effect of cinnamon alone and herbal mixture versus placebo or control on FT level. As the $\mathrm{I}^{2}$ of $0 \%$ represents no statistic heterogeneity between the studies $(P=0.48)$, a fixed effect model was applied to pool the outcomes. The estimated overall effect size demonstrated a significant difference in the FT level in PCOS patients taking cinnamon alone and herbal mixture supplementation, meaning that taking cinnamon alone and herbal mixture led to a significant lower FT level $(\mathrm{MD}=-5.47 \mathrm{pg} / \mathrm{mL}, 95 \% \mathrm{CI}:-8.34$ to -2.61 , $P=0.0002$; Figure S1r).
There is just one clinical trial that has investigated antiandrogen effect of spearmint on PCOS is as follows:

Grant et al (13) conducted a study to determine the spearmint efficacy on hirsutism in PCOS cases. This study lasted 30 day randomized controlled trial duration. Forty-two PCOS patients consumed spearmint tea twice a day and compared with a placebo (chamomile tea). At baseline and after intervention, serum androgen and gonadotropin levels were checked. There was a significant decrease in free and total testosterone in spearmint tea group (3.64 (2.67) versus $5.12(2.14), P<0.05)$ and $(0.62$ (0.34) versus $0.81(0.39), P<0.05)$ respectively. Degree of hirsutism according to the modified DQLI (Dermatology Quality of Life Index) significantly reduced $(P<0.05)$.

Ushiroyama et al (23) by quasi-experimental study concluded that rate of ovulation improved in the both groups who were prescribed mixture of herbs that one group's herbal mixture contained cinnamon as well (Table S2). Moreover, RCT of Lai et al (25) had cinnamon in both intervention and control group herbal mixture, so it was excluded from meta-analysis. But it is worth noting that menstrual rate and hirsutism improved significantly (Table S2). Wang et al (24) in their quasi-experimental study did not report any standard deviation (SD) and $\mathrm{p}$ value, so we could not pool any data of this paper in meta-analysis. However, it should be mentioned that after 8 -weeks treatment level of FBS reduced in both cinnamon and placebo group $(P<0.03)$ and level of QUICKI, HOMAIR, OGTT, and insulin improved just in cinnamon group $(P<0.03)$ (Table S1).

\section{Discussion}

This systematic review and meta-analysis investigated the effectiveness of cinnamon alone and herbal mixture supplementation on glycemic control, sex steroids, lipid profiles, and anthropometric indices in patients with PCOS. Therefore, the studies assessed the effect of cinnamon alone and herbal mixture in combination with other treatment in PCOS. This meta-analysis illustrated that cinnamon alone and herbal mixture may lead to an improvement in FBS, fasting insulin, TG, TC, LDL, MDA, TT, and FT levels, but did not affect weight, waist circumference, HOMA-IR, HDL, FSH, and LH. In case of BMI, cinnamon alone significantly decrease this level; however, herbal mixture had not significant effect.

According to the results of this meta-analysis, both herbal mixtures containing cinnamon and cinnamon alone significantly decrease serum level of insulin and FBS; however, in case of HOMA-IR neither cinnamon nor herbal mixture could not improve serum level of it. In fact, a study of herbal mixture done by Ainehchi et al (16), and studies of cinnamon alone which were conducted by Borzoei et al (26), Hajimonfarednejad et al (27), and Salehpour et al (32) in PCOS concluded that HOMA-IR concentration remarkably reduced at the end of treatment; however Kort et al (28) derived that cinnamon increase 
level of HOMA-IR from 2.3 (1.4-2.8) to 2.5 (0.97-3.3), and placebo reduce this factor from $1.8(0.71-3.3)$ to $1.2(0.78-$ $2.6)$, and this makes the final result inverse. So, it seems conducting more trials in this regard.

Hyperinsulinemia as a significant feature of PCOS by enhancing the activity of $\mathrm{P} 450 \mathrm{c}-17 \alpha$, and reducing sex hormone-binding globulin (SHBG) level as well as insulinlike growth factor binding protein-1 (IGFBP-1) boost the androgen secretion through theca-interstitial and stromal cells (36). Furthermore, it is worth noting that disturbance in GnRH pulsatility in PCOS patients not only lead to enhance of LH to FSH, but also responsible for increasing level of LH by abnormal feedback mechanism via ovarian estrogen (37).

Many studies have indicated that hyperglycemia can increase OS through some non-enzymatic mechanism like inducing the intercellular ROS and generation of electrochemical proton gradient produced by mitochondria which result in deviation of OS (38). In addition, an imbalance between oxidant-antioxidant reduce insulin secretion from pancreatic $\beta$ cells, insulin resistance, and ovarian disorders (39). Also, OS species reaction with lipids cause to elevate peroxidation derivation like MDA (40).

In a study to explore the mechanism of cinnamon on improving insulin resistance, cinnamon reduced level of insulin-like growth factors (IGF-I) and increased level of IGFBP in plasma as well as in ovary tissue to show that cinnamon significantly enhanced insulin sensitivity (41). Cinnamon contains procyanidin polyphenol type-A polymers, which by stimulating autophosphorylation of the insulin receptor and inhibiting protein tyrosine phosphatase I improve insulin sensitivity (42). Also, cinnamon extract by activating glycogen synthase and regulating insulin signaling enhances liver glycogen and improves insulin sensitivity (43), and through enhancing glucosidase enzymes and inhibition of intestinal ATPase decreases absorption of glucose in the small intestine epithelium (44). Polyphenolic compounds of cinnamon such as quercetin, rutin, catechin, kaempferol, and catechin have insulin-like activity (45). The mechanism by which cinnamon regulates blood sugar levels was explained in Hafizur et al (46) study who confirmed that oral administration of 5 and $10 \mathrm{mg} / \mathrm{kg}$ cinnamic acid exerts anti-diabetic activity; as well as improves glucose tolerance by stimulating insulin secretion from pancreas islets. Moreover, Cheng et al (47) valued cinnamon extract that by decreasing gene expression phosphoenolpyruvate carboxykinase and glucose-6-phosphatase as two principal regulators of hepatic gluconeogenesis have insulin like and hypoglycemic effect.

Forest plot of this study demonstrate that serum level of TC, LDL, TG reduced more significantly in herbal mixture (15) in comparison with 2 studies $(26,27)$ which consumed cinnamon alone. Also, it is worth noting that both Hajimonfarednejad et al (27) and Borzoei et al (30) research which prescribed $500 \mathrm{mg}$ cinnamon capsule for 3 times a day for 12 and 8 weeks respectively, the serum level of FBS and insulin of PCOS women in Borzoei et al (30) study reduced much more than Hajimonfarednejad et al (27) study; however the study duration was lesser than Hajimonfarednejad et al. From perspective of mechanism, insulin resistance decreases the activity of hormone-sensitive lipase in adipose tissue and prevents hepatic lipase activity in liver, leading to release free fatty acids (48). Moreover, Khan et al (49) approved that by consuming 1-6 g cinnamon a day for 40 days, level of FBS, TG, LDL, TC decreased in people with type 2 diabetes, however changes of HDL were not significant, which is consistent with our systematic review and meta-analysis. Several studies declared that cinnamaldehyde as active components of cinnamon has anti-hyperlipidemic effect by improving lipid metabolism; however, the underlying mechanisms remain elusive (50,51). Also, cinnamon extract by elevating expression of PPARa lowers plasma TG, and cholesterol levels and elevates HDL levels, while by activating PPAR $\gamma$ improves insulin resistance (50).

The meta-analysis of this study demonstrated the FT and TT level of PCOS patient who consumed cinnamon alone or herbal mixture reduced. The main characteristics of PCOS is hyperinsulinemia which promote androgen secretion and disrupt menstrual cyclicity $(4,52,53)$. Dou et al (41) showed that orally administrated cinnamon extract down-regulate testosterone which were consistent with our results.

\section{Strengths and Limitations}

This systematic review and meta-analysis were accomplished by a complete and sensitive search strategy with the collaboration of research librarian. This study focused on the effect of cinnamon, ginger, spearmint and mixture of them on women with PCOS. Although the studies that evaluate the effect of ginger alone on PCOS could not be found, in herbal mixture it was investigated. Due to the adequate number of included studies in this meta-analysis, we could perform sub-group analysis based on taking cinnamon alone compared to placebo for FBS, HOMA-IR, and BMI

Considering the limitations that exist in these papers regarding the high degree of data heterogeneity and the short duration of intervention and small sample size of studies, which may limit our capability to extract inferences on the effect of long-term herbal medicine supplementation, it is suggested that further studies are needed to evaluate the medicinal herb for long duration as well as follow-up patients for 3-6 months because it is required support for safety. It is suggested that more research be made on ginger effects on PCOS, because no clinical trial studies were found in this area. Risk of bias of included articles were high, it is better to design RCTs and quasi-experimental studies with high quality and low risk factors. 


\section{Conclusions}

This meta-analysis showed that cinnamon alone and herbal mixture containing cinnamon and other herbs may lead to an improvement in FBS, fasting insulin, TG, TC, LDL, MDA, TT, and FT serum level, but did not affect weight, waist circumference, HOMA-IR, HDL, FSH, and LH serum levels. However, the potential benefit is evident regardless of dose. Considering the high serum level of glycemic biomarkers and lipid profile in PCOS women in the included studies, it is possible that these women benefit more from cinnamon and herbal mixture supplements. Therefore, it seems that further studies are necessary.

\section{Conflict of Interests}

Authors declare that they have no conflict of interests.

\section{Ethical Issues}

The current Systematic Review and Meta-Analysis study was authorized by the Ethics Committee of Tabriz University of Medical Sciences (code: TBZMED. REC.1394.576) on 26 November 2015, and was registered in the Iranian Registry of Clinical Trials (IRCT201509295563N7) on 9 January 2016.

\section{Financial Support}

This Systematic Review and Meta-Analysis study is part of a Ph.D. thesis was funded by Tabriz University of Medical Sciences, Tabriz, Iran (grant number: 5.4.9505).

\section{Acknowledgments}

We are thankful for the financial support provided for this thesis.

\section{Supplementary Materials}

Supplementary file 1 contains Figure S1 and Tables S1-S3.

\section{References}

1. Szczuko M, Skowronek M, Zapałowska-Chwyć M, Starczewski A. Quantitative assessment of nutrition in patients with polycystic ovary syndrome (PCOS). Rocz Panstw Zakl Hig. 2016;67(4):419-426.

2. Al Awlaqi A, Alkhayat $\mathrm{K}$, Hammadeh ME. Metabolic syndrome and infertility in women. Int J Womens Health Reprod Sci. 2016;4(3):89-95. doi:10.15296/ijwhr.2016.23

3. Dunaif A, Book CB. Insulin Resistance in the Polycystic Ovary Syndrome. In: Draznin B, Rizza R, eds. Clinical Research in Diabetes and Obesity. Vol 15. Totowa, NJ: Humana Press; 1997:249-274. doi:10.1007/978-1-47573906-0_14

4. Azziz R. Androgen excess is the key element in polycystic ovary syndrome. Fertil Steril. 2003;80(2):252-254. doi:10.1016/s0015-0282(03)00735-0

5. Tayyar AT, Kahraman S. Comparison between cycles of the same patients when using recombinant luteinizing hormone + recombinant follicle stimulating hormone (rFSH), human menopausal gonadotropin $+\mathrm{rFSH}$ and rFSH only. Arch Med Sci. 2019;15(3):673-679. doi:10.5114/ aoms.2017.72408
6. Rosencrantz MA, Coffler MS, Haggan A, et al. Clinical evidence for predominance of delta-5 steroid production in women with polycystic ovary syndrome. J Clin Endocrinol Metab. 2011;96(4):1106-1113. doi:10.1210/jc.2010-2200

7. Agarwal A, Allamaneni SS. Role of free radicals in female reproductive diseases and assisted reproduction. Reprod Biomed Online. 2004;9(3):338-347. doi:10.1016/s14726483(10)62151-7

8. Tangvarasittichai S. Oxidative stress, insulin resistance, dyslipidemia and type 2 diabetes mellitus. World J Diabetes. 2015;6(3):456-480. doi:10.4239/wjd.v6.i3.456

9. Khaki A, Bayatmakoo R, Nouri M, Khaki AA. Remedial Effect of Cinnamon zeylanicum on serum anti-oxidants levels in male diabetic Rat. Life Sci J. 2013;10(4s):103-107.

10. Khodaeifar F, Fazljou SMB, Khaki A, Torbati M, Ouladsahebmadarek E, Khaki AA, et al. Investigating the Role of Hydroalcoholic Extract of Apium graveolens and Cinnamon zeylanicum on Metabolically Change and Ovarian Oxidative Injury in a Rat Model of Polycystic Ovary Syndrome. Int J Womens Health Reprod Sci. 2019;7(1):9298. doi:10.15296/ijwhr.2019.15

11. Jafarnejad S, Keshavarz SA, Mahbubi S, et al. Effect of ginger (Zingiber officinale) on blood glucose and lipid concentrations in diabetic and hyperlipidemic subjects: a meta-analysis of randomized controlled trials. J Funct Foods. 2017;29:127-134. doi:10.1016/j.jff.2016.12.006

12. Khaki A, Khaki AA, Hajhosseini L, Golzar FS, Ainehchi $\mathrm{N}$. The anti-oxidant effects of ginger and cinnamon on spermatogenesis dys-function of diabetes rats. Afr J Tradit Complement Altern Med. 2014;11(4):1-8. doi:10.4314/ ajtcam.v11i4.1

13. Grant P. Spearmint herbal tea has significant anti-androgen effects in polycystic ovarian syndrome. A randomized controlled trial. Phytother Res. 2010;24(2):186-188. doi:10.1002/ptr.2900

14. Abasian Z, Rostamzadeh A, Mohammadi M, Hosseini M, Rafieian-Kopaei M. A review on role of medicinal plants in polycystic ovarian syndrome: pathophysiology, neuroendocrine signaling, therapeutic status and future prospects. Middle East Fertil Soc J. 2018;23(4):255-262. doi:10.1016/j.mefs.2018.04.005

15. Ainehchi N, Khaki A, Ouladsahebmadarek E, et al. The Effect of Clomiphene Citrate, Herbal Mixture, and Herbal Mixture along with Clomiphene Citrate on Clinical and Para-clinical Parameters in Infertile Women with Polycystic Ovary Syndrome: A Randomized Controlled Clinical Trial. Arch Med Sci. 2020; In Press.

16. Ainehchi N, Khaki A, Farshbaf-Khalili A, Hammadeh M, Ouladsahebmadarek E. The Effectiveness of Herbal Mixture Supplements with and without Clomiphene Citrate in Comparison to Clomiphene Citrate on Serum Antioxidants and Glycemic Biomarkers in Women with Polycystic Ovary Syndrome Willing to be Pregnant: A Randomized Clinical Trial. Biomolecules. 2019;9(6). doi:10.3390/biom9060215

17. Ainehchi N, Zahedi A. Effects of Artemisia lanata Extract on Reproductive Parameters of Female Rats. Crescent J Med Biol Sci. 2014;1(2):49-53.

18. Green S, Higgins J, Alderson P, Clarke M, Mulrow C. Cochrane handbook for systematic reviews of interventions. West Sussex, England: John Wiley \& Sons Ltd; 2008.

19. Moher D, Liberati A, Tetzlaff J, Altman DG. Preferred reporting items for systematic reviews and meta-analyses: the PRISMA statement. Ann Intern Med. 2009;151(4):264- 
269, w264. doi:10.7326/0003-4819-151-4-20090818000135

20. Phan K, Tian DH, Cao C, Black D, Yan TD. Systematic review and meta-analysis: techniques and a guide for the academic surgeon. Ann Cardiothorac Surg. 2015;4(2):112122. doi:10.3978/j.issn.2225-319X.2015.02.04

21. Higgins JP, Altman DG, Gøtzsche PC, et al. The Cochrane Collaboration's tool for assessing risk of bias in randomised trials. BMJ. 2011;343:d5928. doi:10.1136/bmj.d5928

22. Fu R, Vandermeer BW, Shamliyan TA, et al. Handling continuous outcomes in quantitative synthesis. In Methods Guide for Effectiveness and Comparative Effectiveness Reviews [Internet]. Rockville (MD): Agency for Healthcare Research and Quality (US); 2013.

23. Ushiroyama T, Hosotani T, Mori K, Yamashita Y, Ikeda A, Ueki M. Effects of switching to wen-jing-tang (unkeito) from preceding herbal preparations selected by eightprinciple pattern identification on endocrinological status and ovulatory induction in women with polycystic ovary syndrome. Am J Chin Med. 2006;34(2):177-187. doi:10.1142/s0192415x06003746

24. Wang JG, Anderson RA, Graham GM 3rd, et al. The effect of cinnamon extract on insulin resistance parameters in polycystic ovary syndrome: a pilot study. Fertil Steril. 2007;88(1):240-243. doi:10.1016/j.fertnstert.2006.11.082

25. Lai L, Flower A, Prescott P, Wing T, Moore M, Lewith G. Standardised versus individualised multiherb Chinese herbal medicine for oligomenorrhoea and amenorrhoea in polycystic ovary syndrome: a randomised feasibility and pilot study in the UK. BMJ Open. 2017;7(2):e011709. doi:10.1136/bmjopen-2016-011709

26. Borzoei A, Rafraf M, Niromanesh S, Farzadi L, Narimani F, Doostan F. Effects of cinnamon supplementation on antioxidant status and serum lipids in women with polycystic ovary syndrome. J Tradit Complement Med. 2018;8(1):128-133. doi:10.1016/j.jtcme.2017.04.008

27. Hajimonfarednejad M, Nimrouzi M, Heydari M, Zarshenas MM, Raee MJ, Jahromi BN. Insulin resistance improvement by cinnamon powder in polycystic ovary syndrome: a randomized double-blind placebo controlled clinical trial. Phytother Res. 2018;32(2):276-283. doi:10.1002/ptr.5970

28. Kort DH, Lobo RA. Preliminary evidence that cinnamon improves menstrual cyclicity in women with polycystic ovary syndrome: a randomized controlled trial. Am J Obstet Gynecol. 2014;211(5):487.e481-486. doi:10.1016/j. ajog.2014.05.009

29. Khan AA, Begum W. Efficacy of Darchini in the management of polycystic ovarian syndrome: a randomized clinical study. J Herb Med. 2019;15:100249. doi:10.1016/j. hermed.2018.11.005

30. Borzoei A, Rafraf M, Asghari-Jafarabadi M. Cinnamon improves metabolic factors without detectable effects on adiponectin in women with polycystic ovary syndrome. Asia Pac J Clin Nutr. 2018;27(3):556-563. doi:10.6133/ apjcn.062017.13

31. Parseh S, Shakerian S, Alizadeh AA. Effect of Chronic Aerobic/Resistive Exercises with Supplementation of Cinnamon on Insulin Resistance in Women with Polycystic Ovary Syndrome in Ahvaz City in 2017. Journal of Arak University of Medical Sciences. 2019;22(1):15-26. [Persian].

32. Salehpour S, Setavand S, Onsori S. A Double-Blind, Placebo-Controlled Comparison of Cinnamon Extract to Metformin Effects upon Insulin Resistance, Apolipoprotein
B: Apolipoprotein A1 Ratio, and BMI of Obese Adolescent Girls with Polycystic Ovary Syndrome. 54th Annual ESPE. European Society for Paediatric Endocrinology; 2015.

33. Wiweko B, Susanto CA. The effect of metformin and cinnamon on serum anti-mullerian hormone in women having PCOS: A Double-blind, randomized, controlled trial. J Hum Reprod Sci. 2017;10(1):31-36. doi:10.4103/jhrs. JHRS_90_16

34. Arentz S, Smith CA, Abbott J, Bensoussan A. Nutritional supplements and herbal medicines for women with polycystic ovary syndrome; a systematic review and metaanalysis. BMC Complement Altern Med. 2017;17(1):500. doi:10.1186/s12906-017-2011-x

35. Talaat B, Ammar IMM. The added value of cinnamon to metformin in controlling symptoms of polycystic ovary syndrome, a randomized controlled trial. Middle East Fertil Soc J. 2018;23(4):440-445.

36. Abtahi-Eivari SH, Moghimian M, Soltani M, Shoorei $\mathrm{H}$, Asghari R, Hajizadeh $\mathrm{H}$, et al. The effect of Galega officinalis on hormonal and metabolic profile in a rat model of polycystic ovary syndrome. Int J Womens Health Reprod Sci. 2018;6(3):276-282. doi: 10.15296/ijwhr.2018.46

37. Alnakash AH, Al-Tae'e NK. Polycystic ovarian syndrome: the correlation between the LH/FSH ratio and disease manifestations. Middle East Fertil Soc J. 2007;12(1):35-40.

38. Nishikawa T, Edelstein D, Du XL, et al. Normalizing mitochondrial superoxide production blocks three pathways of hyperglycaemic damage. Nature. 2000;404(6779):787790. doi:10.1038/35008121

39. Zuo T, Zhu M, Xu W. Roles of oxidative stress in polycystic ovary syndrome and cancers. Oxid Med Cell Longev. 2016;2016:8589318. doi:10.1155/2016/8589318

40. Deepika ML, Nalini S, Maruthi G, et al. Analysis of oxidative stress status through MN test and serum MDA levels in PCOS women. Pak J Biol Sci. 2014;17(4):574-577. doi:10.3923/pjbs.2014.574.577

41. Dou L, Zheng Y, Li L, et al. The effect of cinnamon on polycystic ovary syndrome in a mouse model. Reprod Biol Endocrinol. 2018;16(1):99. doi:10.1186/s12958-018-0418-y

42. Goodyear LJ, Giorgino F, Sherman LA, Carey J, Smith RJ, Dohm GL. Insulin receptor phosphorylation, insulin receptor substrate-1 phosphorylation, and phosphatidylinositol 3-kinase activity are decreased in intact skeletal muscle strips from obese subjects. J Clin Invest. 1995;95(5):2195-2204. doi:10.1172/jci117909

43. Couturier K, Qin B, Batandier C, et al. Cinnamon increases liver glycogen in an animal model of insulin resistance. Metabolism. 2011;60(11):1590-1597. doi:10.1016/j. metabol.2011.03.016

44. Adisakwattana S, Lerdsuwankij O, Poputtachai U, Minipun A, Suparpprom C. Inhibitory activity of cinnamon bark species and their combination effect with acarbose against intestinal alpha-glucosidase and pancreatic alpha-amylase. Plant Foods Hum Nutr. 2011;66(2):143-148. doi:10.1007/ s11130-011-0226-4

45. Anderson RA, Broadhurst CL, Polansky MM, et al. Isolation and characterization of polyphenol type-A polymers from cinnamon with insulin-like biological activity. J Agric Food Chem. 2004;52(1):65-70. doi:10.1021/jf034916b

46. Hafizur RM, Hameed A, Shukrana M, et al. Cinnamic acid exerts anti-diabetic activity by improving glucose tolerance in vivo and by stimulating insulin secretion in vitro. Phytomedicine. 2015;22(2):297-300. doi:10.1016/j. 
phymed.2015.01.003

47. Cheng DM, Kuhn P, Poulev A, Rojo LE, Lila MA, Raskin I. In vivo and in vitro antidiabetic effects of aqueous cinnamon extract and cinnamon polyphenol-enhanced food matrix. Food Chem. 2012;135(4):2994-3002. doi:10.1016/j. foodchem.2012.06.117

48. Ormazabal V, Nair S, Elfeky O, Aguayo C, Salomon C, Zuniga FA. Association between insulin resistance and the development of cardiovascular disease. Cardiovasc Diabetol. 2018;17(1):122. doi:10.1186/s12933-018-0762-4

49. Khan A, Safdar M, Ali Khan MM, Khattak KN, Anderson RA. Cinnamon improves glucose and lipids of people with type 2 diabetes. Diabetes Care. 2003;26(12):3215-3218. doi:10.2337/diacare.26.12.3215

50. Huang B, Yuan HD, Kim DY, Quan HY, Chung SH. Cinnamaldehyde prevents adipocyte differentiation and adipogenesis via regulation of peroxisome proliferatoractivated receptor-gamma (PPARgamma) and AMPactivated protein kinase (AMPK) pathways. J Agric Food
Chem. 2011;59(8):3666-3673. doi:10.1021/jf104814t

51. Sheng X, Zhang Y, Gong Z, Huang C, Zang YQ. Improved insulin resistance and lipid metabolism by cinnamon extract through activation of peroxisome proliferatoractivated receptors. PPAR Res. 2008;2008:581348. doi:10.1155/2008/581348

52. Nestler JE, Jakubowicz DJ, de Vargas AF, Brik C, Quintero $\mathrm{N}$, Medina F. Insulin stimulates testosterone biosynthesis by human thecal cells from women with polycystic ovary syndrome by activating its own receptor and using inositolglycan mediators as the signal transduction system. J Clin Endocrinol Metab. 1998;83(6):2001-2005. doi:10.1210/jcem.83.6.4886

53. Hajhosseini L, Khaki A, Merat E, Ainehchi N. Effect of Rosmarinic Acid on Sertoli Cells Apoptosis and Serum Antioxidant Levels in Rats After Exposure to Electromagnetic Fields. Afr J Tradit Complement Altern Med. 2013;10(6):477-80.

(c) 2019 The Author (s); This is an open-access article distributed under the terms of the Creative Commons Attribution License (http://creativecommons.org/licenses/by/4.0), which permits unrestricted use, distribution, and reproduction in any medium, provided the original work is properly cited. 